\title{
Spinal anesthesia for emergency cesarean section in a preeclampsia patient diagnosed with type 1 neurofibromatosis
}

\author{
Wang Yong Lee, Yong Sup Shin, Chae Seong Lim, Woo Suk Chung, and Byung Muk Kim \\ Department of Anesthesiology and Pain Medicine, School of Medicine, Chungnam National University, Daejeon, Korea
}

Type 1 neurofibromatosis, also referred to as von Recklinghausen's disease, is characterized by café-au-lait spots and benign cutaneous neurofibromas and is more common than type 2 neurofibromatosis $[1,2]$. In contrast, type 2 neurofibromatosis affects the central nervous system (CNS) via spinal cord tumors and bilateral vestibular schwannomas [1-3]. Due to the involvement with the CNS, regional anesthesia in type 2 neurofibromatosis without careful preoperative examination can be extremely dangerous and many anesthesiologists prefer general anesthesia [1]. On the other hand, regional anesthesia could be useful in type 1 neurofibromatosis because CNS involvement is rare. We report a successful case of spinal anesthesia in a type 1 neurofibromatosis patient diagnosed with preeclampsia during an emergency cesarean section.

A 32-year-old pregnant woman (height $163 \mathrm{~cm}$, weight 72 $\mathrm{kg}, 41+4$ weeks) was transferred to our hospital due to fetal distress. Although there was no specific event during the patients' prenatal care, the patient developed preeclampsia with high blood pressure and severe generalized edema at $41+2$ weeks. Past medical history revealed neurofibromatosis, which was diagnosed 12 years ago with the characteristics of café-aulait spots and benign cutaneous neurofibromas. The patient had no history of neither general nor regional anesthesia, although neurofibroma excision was performed under local anesthesia several times.

Fetal distress was increased during pharmacologic labor induction and the patient was scheduled for cesarean section. The patient arrived at the operating room with a four-hour fasting state and without premedication. There were no neurological symptoms and no mass was observed in the tongue, pharynx and larynx. Preoperative laboratory tests were non-specific, except proteinuria $(2+)$. Preoperative systolic blood pressure was relatively highly, being measured at $140-170 \mathrm{mmHg}$. Hartmann's solution was given via peripheral intravenous line and $\mathrm{MgSO}_{4}$ was administered. Although we could not completely rule out spinal cord neurofibroma without computerized tomography or magnetic resonance imaging, spinal anesthesia was performed considering the negative neurological symptoms and the characteristic clinical features of type 1 neurofibromatosis. The patient was positioned as left lateral decubitus. Spinal anesthesia was first performed at the L4-5 level without success. After the second attempt, the level was changed to L3-4 and 0.5\% heavy bupivacaine $10 \mathrm{mg}$ was administered after cerebrospinal fluid was confirmed by a paramedian approach. Due to several fibromas in the skin puncture site, we were not able to perform a medial approach (Fig. 1). Sensory block level was confirmed as T4 dermatome by ice and operation was started. Total induction time was approximately 30 minutes and vital signs were stable with no specific event during the operation. The patient was discharged 6 days later without any complications.

Neurofibromatosis is a disease which involves the nervous system and skin. Neurofibromatosis is an inherited autosomal dominant disease and classified as type 1 and type 2. It is caused by a mutation of different chromosomes, type 1 in chromosome 17 q11.2 and type 2 in chromosome 22q12.1 [3]. Type 1 neurofibromatosis (von Recklinghausen's disease) is characterized by skin lesions, such as benign neurofibromas of the skin (cutaneous neurofibroma) and coffee like color spots (café-au-lait spot) [4].

Corresponding author: Yong Sup Shin, M.D., Ph.D., Department of Anesthesiology and Pain Medicine, School of Medicine, Chungnam National University, 33, Munhwa-ro, Jung-gu, Daejeon 301-721, Korea. Tel: 82-42-280-7840, Fax: 82-42-280-7968, E-mail: ysshin@cnu.ac.kr (c) This is an open-access article distributed under the terms of the Creative Commons Attribution Non-Commercial License (http:// creativecommons.org/licenses/by-nc/3.0/), which permits unrestricted non-commercial use, distribution, and reproduction in any medium, provided the original work is properly cited. 


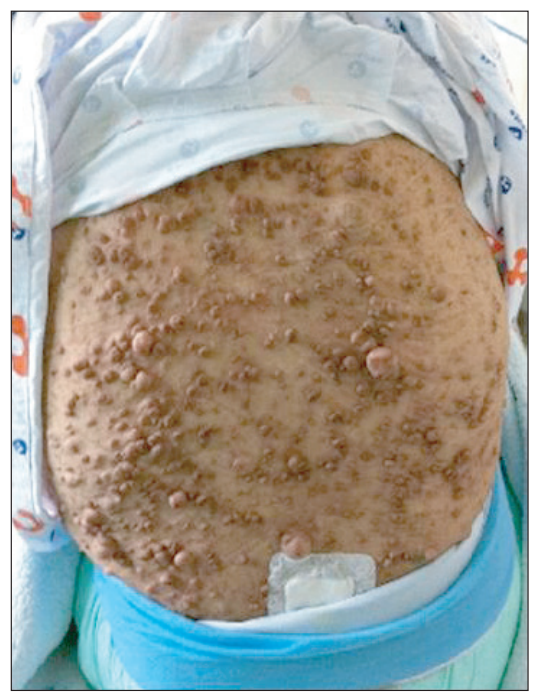

Fig. 1. Parturient's tumor, sized $1-2 \mathrm{~cm}$, was spread throughout the entire body as well as the back.

Unlike type 2 neurofibromatosis, it is also known to be associated with pheochromocytoma [3]. Type 2 neurofibromatosis often invades the central nervous system; moreover, the main clinical feature is bilateral vestibular schwannomas leading to gradual hearing loss [3]. Other clinical features, such as meningioma of brain, schwannoma of cranial, spinal or peripheral nerve and juvenile cortical cataract, can also exist [1]. Neurofibromatosis parturients are known to be associated with hypertension, HELLP syndrome, spontaneous abortion and increasing of number and size of neurofibromas [3]. The size of CNS tumors in neurofibromatosis patients can also rapidly increase during pregnancy [5]. Performing regional anesthesia in patients with CNS involvement needs extensive examination. A spinal tumor may exist in the injection level or tumor cells can be spread into the CNS by spinal and epidural needles. Such risk factors make general anesthesia appear more advisable, but other risk factors also exist. Difficulty of airway management is a known major cause of anesthesia-related maternal deaths. In neurofibromatosis patients, additional masses in the tongue, pharynx and larynx may interfere with intubation. Anesthesiologists should perform a careful review of the patient's airway (Cormack \& Lehane grade, past medical history and Mallampati class, etc.). If airway management seems difficult, intubation in the awake state or fiberoptic bronchoscopy may be necessary. Other risk factors of general anesthesia in hypertensive parturients include intracranial hemorrhage or pulmonary edema due to extensive increase of blood pressure during tracheal intu-, extubation as well as longer effects of muscle relaxants, if $\mathrm{MgSO}_{4}$ was used preoperatively.

In our present case, preoperative radiographic examination was not available. Although this makes regional anesthesia complicating, the patient showed classical features of type 1 neurofibromatosis and had no neurological symptoms. Also, the patient's fasting time was only 4 hours and $\mathrm{MgSO}_{4}$ was preoperatively used. The high risk of aspiration also makes regional anesthesia favorable. The patient's entire body was covered with neurofibromas, including the needle puncture site used for the median approach. However, by using a paramedian approach, spinal anesthesia was successfully performed without any complications to the mother and neonate.

In conclusion, we present a case of successful spinal anesthesia during an emergency cesarean section in a neurofibromatosis patient without extensive neural evaluation. Anesthesiologists should consider the complex and diverse factors that exist in neurofibromatosis parturients in order to provide optimal anesthesia.

\section{References}

1. Hirsch NP, Murphy A, Radcliffe JJ. Neurofibromatosis: clinical presentations and anaesthetic implications. Br J Anaesth 2001; 86: 555-64.

2. Spiegel R, Mächler M, Stocker HP, Boltshauser E, Schmid W. Neurofibromatosis Type 1: genetic studies with DNA markers in 38 families. Schweiz Med Wochenschr 1991; 121: 1445-52.

3. Spiegel JE, Hapgood A, Hess PE. Epidural anesthesia in a parturient with neurofibromatosis type 2 undergoing cesarean section. Int J Obstet Anesth 2005; 14: 336-9.

4. Syrbe S, Eberle K, Strenge S, Bernhard MK, Herbertz S, Bierbach U, et al. Neurofibromatosis type 1 and associated clinical abnormalities in 27 children. Klin Padiatr 2007; 219: 326-32.

5. Segal D, Holcberg G, Sapir O, Sheiner E, Mazor M, Katz M. Neurofibromatosis in pregnancy. Maternal and perinatal outcome. Eur J Obstet Gynecol Reprod Biol 1999; 84: 59-61. 